Article

\title{
Effect of Handgrip Strength on Clinical Outcomes of Patients with Hepatocellular Carcinoma Treated with Lenvatinib
}

\author{
Yurika Kotoh ${ }^{1}$, Issei Saeki ${ }^{1, *(1)}$, Takahiro Yamasaki ${ }^{2, * \mathbb{D}}$, Ryo Sasaki ${ }^{1}$, Norikazu Tanabe ${ }^{1}$, \\ Takashi Oono ${ }^{1}$, Takashi Matsuda ${ }^{1}$, Takuro Hisanaga ${ }^{1,3}$, Toshihiko Matsumoto ${ }^{1,2}$, Isao Hidaka ${ }^{1}$, \\ Tsuyoshi Ishikawa ${ }^{1}$, Taro Takami ${ }^{1} \mathbb{D}$ and Isao Sakaida ${ }^{1}$ \\ 1 Department of Gastroenterology and Hepatology, Yamaguchi University Graduate School of Medicine, Ube, \\ Yamaguchi 755-8505, Japan; g030ub@yamaguchi-u.ac.jp (Y.K.); ryo0530@yamaguchi-u.ac.jp (R.S.); \\ norikazu@yamaguchi-u.ac.jp (N.T.); toono@yamaguchi-u.ac.jp (T.O.); t.mazda@yamaguchi-u.ac.jp (T.M.); \\ t-hisa01@yamaguchi-u.ac.jp (T.H.); tm0831@yamaguchi-u.ac.jp (T.M.); isao-h@yamaguchi-u.ac.jp (I.H.); \\ tsu0920@yamaguchi-u.ac.jp (T.I.); t-takami@yamaguchi-u.ac.jp (T.T.); sakaida@yamaguchi-u.ac.jp (I.S.) \\ 2 Department of Oncology and Laboratory Medicine, Yamaguchi University Graduate School of Medicine, \\ Ube, Yamaguchi 755-8505, Japan \\ 3 Department of Medical Education, Yamaguchi University Graduate School of Medicine, Ube, \\ Yamaguchi 755-8505, Japan \\ * Correspondence: issaeki@yamaguchi-u.ac.jp (I.S.); t.yama@yamaguchi-u.ac.jp (T.Y.); \\ Tel.: +81-836-22-2241 (I.S.); +81-836-22-2336 (T.Y.)
}

Received: 7 July 2020; Accepted: 3 August 2020; Published: 5 August 2020

\begin{abstract}
Previous studies have reported prognostic factors for hepatocellular carcinoma (HCC) patients receiving lenvatinib; however, no studies have evaluated the effects of both handgrip strength and skeletal muscle mass on the clinical outcomes. Therefore, this retrospective study investigated the individual effect of handgrip strength, skeletal muscle mass, and sarcopenia on clinical outcomes of 53 HCC patients treated with lenvatinib. Before receiving lenvatinib, handgrip strength and skeletal muscle index (SMI) were measured. Low handgrip strength and muscle depletion were defined as $<26$ and $<18 \mathrm{~kg}$ and SMI $<42$ and SMI $<38 \mathrm{~cm}^{2} / \mathrm{m}^{2}$ in men and women, respectively. Sarcopenia was defined as having low handgrip strength and muscle depletion. Multivariate analysis identified modified albumin-bilirubin grade 1-2a $(p=0.010)$, Barcelona Clinic Liver Cancer stage A-B $(p=0.011)$, and absence of low handgrip strength $(p=0.015)$ as favorable prognostic factors for survival. Furthermore, sarcopenia was an independent significant prognostic factor for survival. Time to treatment failure was associated with handgrip strength and sarcopenia. Our findings suggest that handgrip strength may be a useful marker of clinical outcomes in HCC patients treated with lenvatinib.
\end{abstract}

Keywords: hepatocellular carcinoma; handgrip strength; lenvatinib; skeletal muscle; survival; time to treatment failure

\section{Introduction}

Sarcopenia was initially defined by Rosenberg as age-related loss of muscle mass and function [1,2]; however, sarcopenia caused by various factors, such as chronic liver diseases including liver cirrhosis (LC) and hepatocellular carcinoma (HCC), has recently become an important concern [3]. As the definition of sarcopenia varies among various regions, such as Europe and Asia [3,4], the specific definition should be applied to each region. In 2015, the Japan Society of Hepatology (JSH) proposed sarcopenia criteria for patients with chronic liver disease [5], which consists of measurements for 
handgrip strength and skeletal muscle mass by computed tomography $(\mathrm{CT})$ or bioelectrical impedance analysis (BIA). Sarcopenia has been reported to be associated with the prognosis of patients with LC or HCC [6-8]. For HCC patients receiving sorafenib, the absence of skeletal muscle depletion is a favorable predictor of survival [9-12]. Another report showed that muscle depletion predicted the occurrence of sorafenib-related toxicities [13]. However, the measurement of skeletal muscle mass using $\mathrm{CT}$ alone without the assessment of handgrip strength has been used as the definition of sarcopenia in most previous reports [6-13].

The recent success of a clinical trial, phase III REFLECT study, has changed the treatment strategy in advanced HCC patients [14]. The REFLECT study demonstrated that lenvatinib was non-inferior to sorafenib regarding overall survival (OS) and had a strong objective response (OR) and different adverse events (AEs) compared with sorafenib [15]. Therefore, lenvatinib is also considered as a first-line agent in addition to sorafenib [16-19]. Recently, a subanalysis of the REFLECT study demonstrated the efficacy of sequential therapy (most common being sorafenib) [20]. Therefore, it is important to use lenvatinib for a longer duration in responders and to switch to another molecular targeted agent (MTA) earlier in non-responders to obtain a favorable OS. Although previous studies have reported prognostic factors for HCC patients receiving lenvatinib [21-26], no studies have evaluated the effects of both handgrip strength and skeletal muscle mass on the clinical outcomes. Therefore, this retrospective study aimed to investigate the individual effect of handgrip strength, skeletal muscle mass, and sarcopenia on clinical outcomes of HCC patients treated with lenvatinib.

\section{Methods}

\subsection{Study Cohort}

This retrospective cohort study included 59 HCC patients who were administered lenvatinib between May 2018 and January 2020. HCC diagnosis was based on pathological or imaging confirmatory findings. Patients were excluded if skeletal muscle mass and handgrip strength were not measured before the treatment initiation. Following exclusion, a total of 53 patients were enrolled in this study. The last observation date was set as 30 April 2020.

The study protocol (H26-109) was approved by the Institutional Review Board of Yamaguchi University Hospital and was conducted in compliance with the ethical principles of the Declaration of Helsinki.

\subsection{Treatment and Follow-up Strategy}

Lenvatinib (Lenvima ${ }^{\circledR}$; Eisai Co., Ltd., Tokyo, Japan) was started with $12 \mathrm{mg}$ or $8 \mathrm{mg}$ doses based on body weight and liver function. Patients with Child-Pugh A function and weighing $\geq 60 \mathrm{~kg}$ received $12 \mathrm{mg}$ and those weighing $<60 \mathrm{~kg}$ and/or with Child-Pugh B received $8 \mathrm{mg}$. The drug dose was tapered and interrupted based on AEs. Lenvatinib was administered until radiological tumor progression or onset of intolerable AEs.

\subsection{Evaluation of Handgrip Strength and Skeletal Muscle Mass}

Handgrip strength was assessed with a grip strength dynamometer (T.K.K. 5401; Takei Scientific Instruments Co., Ltd., Niigata, Japan). Low handgrip strength was defined as $<26 \mathrm{~kg}$ in men and $<18 \mathrm{~kg}$ in women according to the JSH criteria [5].

Skeletal muscle mass was measured using CT images of the third lumbar vertebra level $[5,9]$. This analysis was performed using an AZE 3D workstation (AZE Virtual Place Raijin; AZE ltd., Tokyo, Japan). Skeletal muscle mass was expressed as skeletal muscle index (SMI), calculated as skeletal muscle mass divided by height squared $\left(\mathrm{cm}^{2} / \mathrm{m}^{2}\right)$. According to the JSH criteria, muscle depletion was defined as SMI $<42 \mathrm{~cm}^{2} / \mathrm{m}^{2}$ and SMI $<38 \mathrm{~cm}^{2} / \mathrm{m}^{2}$ in men and women, respectively [5].

The diagnosis of sarcopenia was made using the JSH diagnostic guideline for sarcopenia in patients with liver disease [5]. According to these criteria, sarcopenia is defined as having low handgrip 
strength ( $<26 \mathrm{~kg}$ in men and $<18 \mathrm{~kg}$ in women) and muscle depletion $\left(\mathrm{SMI}<42 \mathrm{~cm}^{2} / \mathrm{m}^{2}\right.$ in men and $<38 \mathrm{~cm}^{2} / \mathrm{m}^{2}$ in women).

\subsection{Evaluation of the Treatment Response and Liver Function}

Treatment response was evaluated by dynamic $\mathrm{CT}$ or magnetic resonance imaging according to the modified response evaluation criteria in solid tumors [27]. Tumor assessment was performed every 2-3 months. OR, defined as the complete response (CR) or partial response (PR), and disease control (DC), defined as CR, PR, or stable disease (SD), were evaluated. A case of death before conducting the tumor assessment was defined as no evaluation (NE). Liver function was evaluated by using the Child-Pugh classification and modified albumin-bilirubin (mALBI) grade [28].

\subsection{Statistical Analysis}

Continuous variables are expressed as the median and interquartile range and compared using the Wilcoxon test. Categorical variables were analyzed by the chi-squared test, Cochran-Armitage trend test, or Fisher's exact test. The OS and duration of lenvatinib therapy (time to treatment failure (TTF)) were estimated by the Kaplan-Meier method and compared with the log-rank test. TTF was defined as the interval between the induction and the discontinuation time of lenvatinib. Survival time was defined as the duration between the induction of lenvatinib and the last follow-up or death. The follow-up period ended on 30 April 2020. Univariate and multivariate Cox proportional hazard models were used to calculate hazard ratio (HR) for OS and their $95 \%$ confidence intervals (CIs). A $p$-value of $<0.05$ was considered statistically significant. All statistical analyses were performed using the JMP Pro 14 (SAS Institute Inc., Cary, NC, USA).

\section{Results}

\subsection{Patient Characteristics}

Patient profiles are summarized in Table 1. The median age was 72.0 years, and there were 41 men and 12 women. The etiology of HCC was hepatitis B virus, hepatitis virus C, and others in 7, 20, and 26 patients, respectively. We observed that 42 and 11 patients, respectively, had findings of Child-Pugh class A and B, whereas 5, 13, 31, and 4 patients were classified as having mALBI grade [28] 1, 2a, 2b, and 3, respectively. According to the Barcelona Clinic Liver Cancer (BCLC) staging system [29], 1 patient was classified as stage $A, 27$ as stage $B$, and 25 as stage $C$. As one patient with BCLC stage A was not an adaptation of local therapy (ablation and transcatheter arterial chemoembolization) technically, lenvatinib was administered to this patient. Eleven patients $(20.8 \%)$ had macrovascular invasion (MVI) and 15 (28.3\%) had extrahepatic spread (EHS). The median number and size of tumors were 4.0 and $28.0 \mathrm{~mm}$, respectively.

The median SMIs for male and female patients were 45.8 and $34.1 \mathrm{~cm}^{2} / \mathrm{m}^{2}$, respectively; muscle depletion was observed in 26 of 53 patients (49.1\%). The median handgrip strengths for male and female patients were 28.1 and $15.2 \mathrm{~kg}$, respectively; low handgrip strength was observed in 25 of all patients $(47.2 \%)$. Finally, 15 patients $(28.3 \%)$ were diagnosed with sarcopenia according to the JSH criteria.

Seven patients $(13.2 \%)$ had a history of MTA treatment (sorafenib), while four $(7.5 \%)$ were treatment naive. The median observation period was 9.7 (4.9-15.2) months. 
Table 1. Patient characteristics.

\begin{tabular}{|c|c|c|}
\hline & & $n=53$ \\
\hline Age (year) & & $72.0(68.5-79.5)$ \\
\hline Sex Male/Female & & $41 / 12$ \\
\hline Etiology HBV/HCV/Others & & $7 / 20 / 26$ \\
\hline Child-Pugh class A/B & & $42 / 11$ \\
\hline ALBI score & & $-2.05(-2.35$ to -1.75$)$ \\
\hline mALBI grade $1 / 2 a / 2 b / 3$ & & $5 / 13 / 31 / 4$ \\
\hline BCLC A/B/C/D & & $1 / 27 / 25 / 0$ \\
\hline Tumor number & & $4.0(2.0-10.0)$ \\
\hline Tumor size $(\mathrm{mm})$ & & $28.0(18.0-39.0)$ \\
\hline Macrovascular invasion $+/-$ & & $11 / 42$ \\
\hline Extrahepatic spread +/- & & $15 / 38$ \\
\hline \multirow{2}{*}{ Handgrip (kg) } & M & $28.1(23.2-32.7)$ \\
\hline & $\mathrm{F}$ & $15.2(12.7-17.6)$ \\
\hline Low handgrip strength $+/-$ & & $25 / 28$ \\
\hline $\mathrm{SMI}\left(\mathrm{cm}^{2} / \mathrm{m}^{2}\right)$ & M & $45.8(39.5-50.7)$ \\
\hline $\sin \left(\mathrm{cm}^{2} / \mathrm{m}^{2}\right)$ & $\mathrm{F}$ & $34.1(33.1-37.4)$ \\
\hline Muscle depletion +/- & & $26 / 27$ \\
\hline Sarcopenia +/- & & $15 / 38$ \\
\hline
\end{tabular}

HBV, hepatitis B virus; HCV, hepatitis C virus; ALBI score, albumin-bilirubin score; mALBI grade, modified albumin-bilirubin grade; BCLC, Barcelona Clinic Liver Cancer staging system; SMI, skeletal muscle index.

\subsection{Treatment Response, Survival, and TTF}

In this study, 1, 20, 17, 7, and 8 patients achieved CR, PR, SD, PD, and NE. The objective response rate and disease control rate were $39.6 \%$ (21/53) and 71.7\% (38/53), respectively. The median survival time (MST) was 24.0 months (Figure 1A), and the median TTF was 4.2 months (Figure 1B).

\section{(A)}

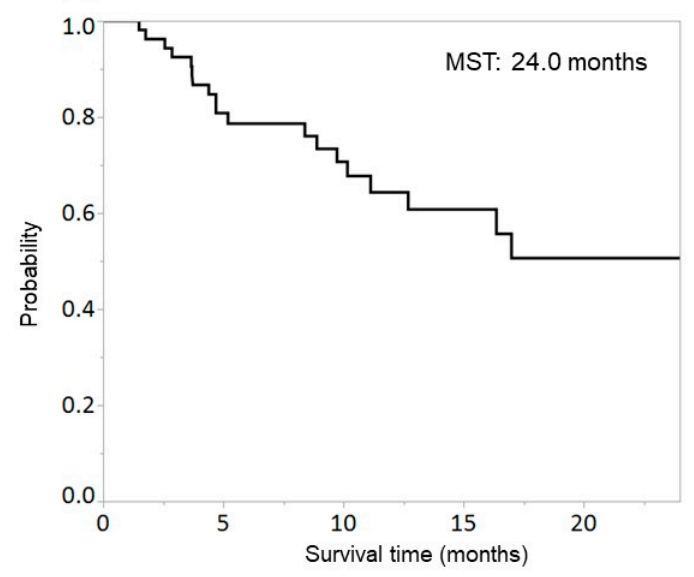

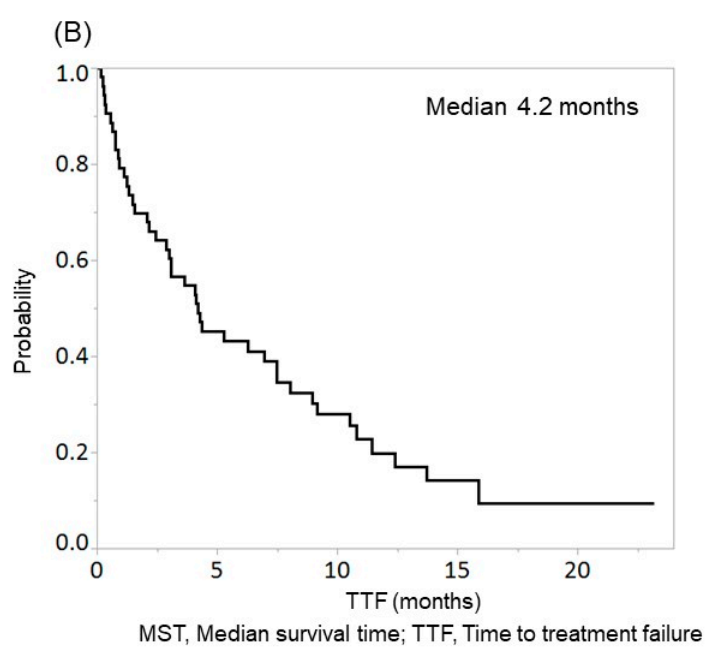

Figure 1. Overall survival (OS) (A) and time to treatment failure (TTF) (B) in patients with hepatocellular carcinoma (HCC) treated with lenvatinib. The median survival time (MST) and median TTF were 24.0 months and 4.2 months, respectively.

\subsection{Survival According to the Evaluation of Handgrip Strength, Skeletal Muscle, or Sarcopenia}

Patients with low handgrip strength showed significantly shorter survival than those with high handgrip strength (MST: low handgrip strength group, 10.2 months; high handgrip strength group, 24.0 months; $p=0.010$; Figure $2 \mathrm{~A}$ ), while no significant difference was found between patients with and without muscle depletion ( $p=0.675$; Figure 2B). Significant differences were observed in age, sex, 
and etiology when patients were evaluated according to the handgrip strength and in age and sex when patients were evaluated according to muscle depletion (Supplementary Table S1).
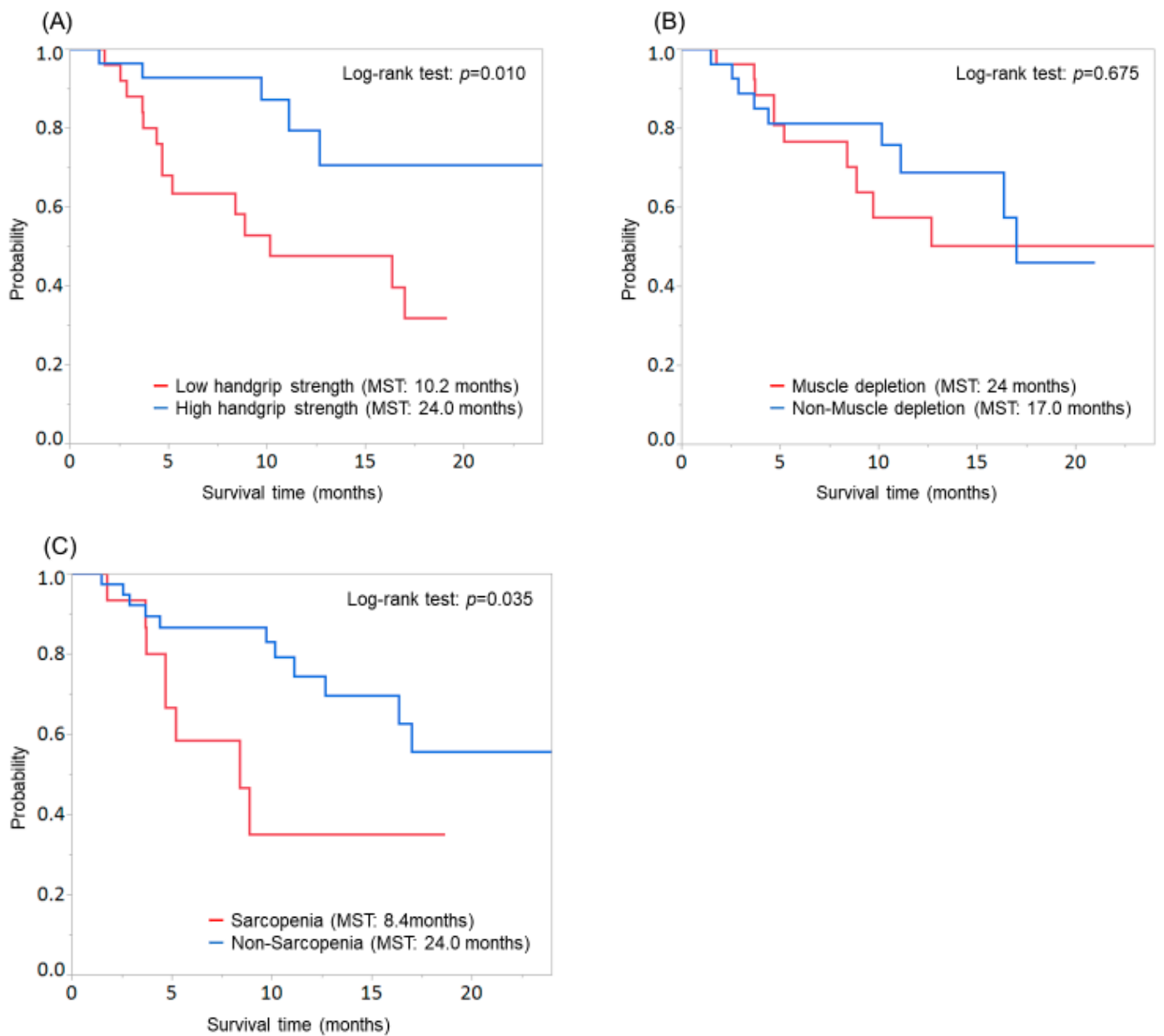

MST, Median survival time

Figure 2. Comparison of overall survival according to the evaluation of handgrip strength, skeletal muscle, or sarcopenia. (A) Patients with low handgrip strength showed significantly shorter survival than those with high handgrip strength (MST: low handgrip strength group, 10.2 months; high handgrip strength group, 24.0 months; $p=0.010$ ). (B) No significant difference was found between patients with and without muscle depletion $(p=0.675)$. (C) Patients with sarcopenia showed significantly shorter survival than those without sarcopenia (MST: sarcopenia group, 8.4 months; non-sarcopenia group, 24.0 months; $p=0.035)$.

Moreover, patients with sarcopenia showed significantly shorter survival than those without sarcopenia (MST: sarcopenia group, 8.4 months; non-sarcopenia group, 24.0 months; $p=0.035$; Figure $2 \mathrm{C}$ ). In the evaluation of patients according to sarcopenia, significant differences were found in age and sex (Supplementary Table S2).

\subsection{Predictive Factors Associated with OS}

Factors assessed in the univariate analysis were age ( $<72$ years $/ \geq 72$ years), sex (male/female), Child-Pugh classification (A/B), mALBI grade (1-2a/2b-3), BCLC stage (A-B/C), muscle depletion (presence/absence), low handgrip strength (presence/absence), and sarcopenia (presence/absence). Of these factors, mALBI grade $(p=0.018)$, BCLC stage $(p=0.007)$, low handgrip strength $(p=0.010)$, and sarcopenia $(p=0.050)$ were significant factors, but muscle depletion was not significant (Table 2A). 
Table 2. Univariate and multivariate analyses of factors associated with overall survival.

\begin{tabular}{|c|c|c|c|c|c|c|c|c|c|}
\hline & \multicolumn{3}{|c|}{ Univariate Analysis (A) } & \multicolumn{3}{|c|}{ Multivariate Analysis (B) } & \multicolumn{3}{|c|}{ Multivariate Analysis (C) } \\
\hline & Hazard ratio & $95 \% \mathrm{CI}$ & $p$-value & Hazard ratio & $95 \%$ CI & $p$-value & Hazard ratio & $95 \% \mathrm{CI}$ & $p$-value \\
\hline Age $<72 / \geq 72$ & 1.580 & $0.634-4.256$ & 0.330 & & & & & & \\
\hline Sex Male/Female & 1.203 & $0.388-3.152$ & 0.728 & & & & & & \\
\hline Child-Pugh class A/B & 1.586 & $0.512-4.151$ & 0.395 & & & & & & \\
\hline mALBI grade $1-2 a / 2 b-3$ & 3.724 & $1.233-16.064$ & 0.018 & 4.209 & $1.370-18.362$ & 0.010 & 5.247 & $1.607-24.201$ & 0.004 \\
\hline BCLC $\mathrm{A}-\mathrm{B} / \mathrm{C}$ & 3.715 & $1.420-11.501$ & 0.007 & 3.480 & $1.320-10.833$ & 0.011 & 3.258 & $1.229-10.179$ & 0.017 \\
\hline Muscle depletion -/+ & 1.214 & $0.487-3.072$ & 0.675 & & & & & & \\
\hline Low handgrip strength $-/+$ & 3.518 & $1.343-10.904$ & 0.010 & 3.313 & $1.257-10.314$ & 0.015 & & & \\
\hline Sarcopenia $-/+$ & 2.621 & $0.999-6.592$ & 0.050 & & & & 3.256 & $1.136-9.478$ & 0.029 \\
\hline
\end{tabular}

mALBI grade, modified albumin-bilirubin grade; BCLC, Barcelona Clinic Liver Cancer staging system. 
As having low handgrip strength was considered in the diagnosis of sarcopenia, multivariate analysis was performed separately using either of the two factors (low handgrip strength and sarcopenia). The first multivariate analysis, which was performed using factors found to be significant ( $p$-value $\leq 0.1 ;$ mALBI grade, BCLC stage, and low handgrip strength), revealed the following three factors as significant: mALBI grade $2 \mathrm{~b}-3$ (HR, $4.209 ; 95 \% \mathrm{CI}, 1.370-18.362 ; p=0.010)$, BCLC stage C (HR, 3.480; 95\% CI, 1.320-10.833; $p=0.011)$, and the presence of low handgrip strength (HR, 3.313; 95\% CI, 1.257-10.314; $p=0.015$; Table 2B). Then, the second multivariate analysis of significant factors ( $p$-value $\leq 0.1 ;$ mALBI grade, BCLC stage, and sarcopenia) revealed these three factors as significant: mALBI grade 2b-3 (HR, 5.247; 95\% CI, 1.607-24.201; $p=0.004)$, BCLC stage C (HR, 3.258; 95\% CI, $1.229-10.179 ; p=0.017)$, and the presence of sarcopenia (HR, 3.256; 95\% CI, 1.136-9.478; $p=0.029$; Table 2C).

\subsection{TTF According to Handgrip Strength, Skeletal Muscle, or Sarcopenia}

Patients with low handgrip strength tended to discontinue the treatment compared with those with high handgrip strength (median TTF: low handgrip strength group, 3.6 months; high handgrip strength group, 6.2 months; $p=0.120$; Figure $3 \mathrm{~A}$ ), while no significant difference was found between patients with and without muscle depletion $(p=0.683$; Figure $3 \mathrm{~B})$. Among patients with low handgrip strength, 2 continued treatment, 4 stopped treatment due to disease progression, and 19 stopped treatment due to AEs. Among patients with high handgrip strength, 8 continued treatment, 8 stopped treatment due to disease progression, and 12 stopped treatment due to AEs. Therefore, patients with low handgrip strength tended to discontinue lenvatinib therapy due to AEs compared with those with high handgrip strength $(p=0.099)$.

In contrast, patients with sarcopenia showed significantly worse TTF than those without sarcopenia (median TTF: sarcopenia group, 3.6 months; non-sarcopenia group, 5.3 months; $p=0.036$; Figure 3C). 
(A)

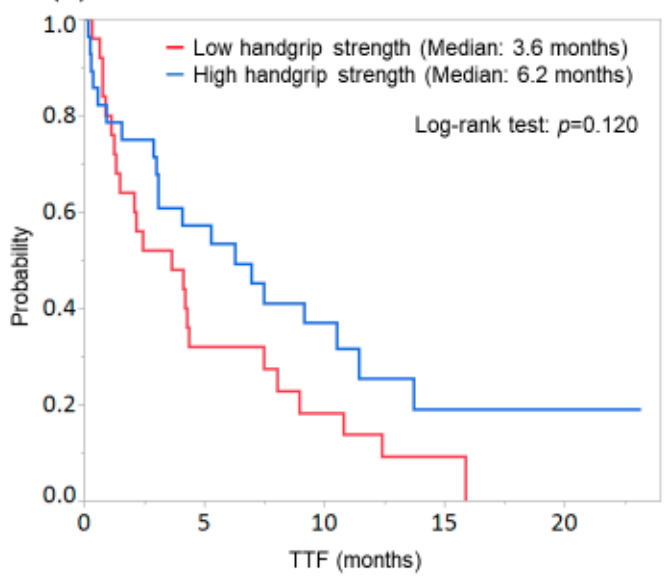

(B)

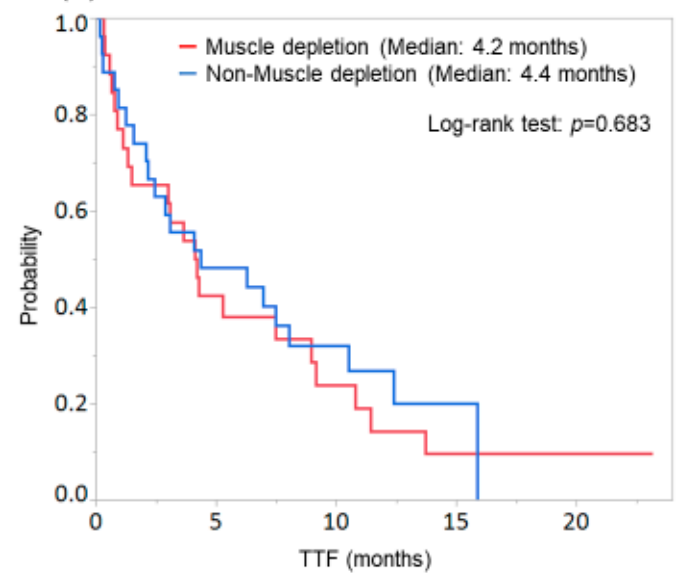

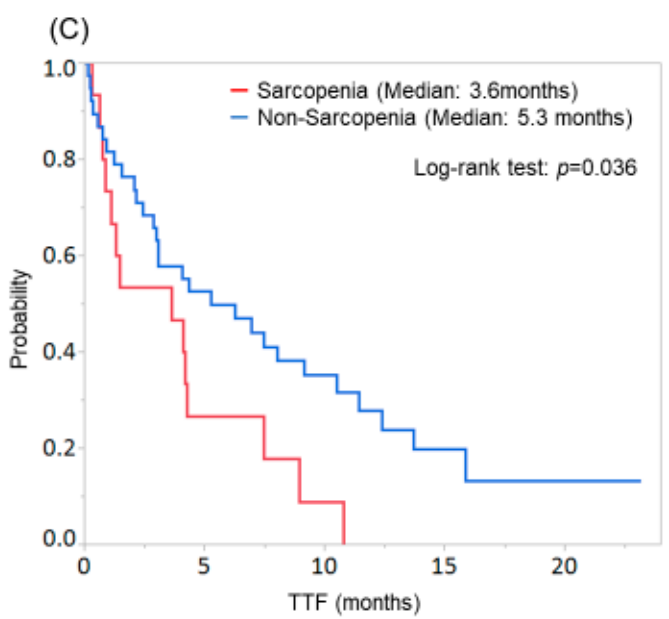

TTF, Time to treatment failure

Figure 3. Comparison of time to treatment failure (TTF) according to the evaluation of handgrip strength, skeletal muscle, or sarcopenia. (A) Patients with low handgrip strength tended to discontinue the treatment than those with high handgrip strength (median TTF: low handgrip strength group, 3.6 months; high handgrip strength group, 6.2 months; $p=0.120$ ). (B) No significant difference was found between patients with and without muscle depletion $(p=0.683)$. (C) Patients with sarcopenia showed significantly worse TTF than those without sarcopenia (median TTF: sarcopenia group, 3.6 months; non-sarcopenia group, 5.3 months; $p=0.036$ ).

\section{Discussion}

Our study focused on HCC patients treated with lenvatinib and, to our knowledge, it is the first to determine the individual effect of handgrip strength and skeletal muscle mass. In our results, patients with low handgrip strength showed significantly shorter survival than those with high handgrip strength, and low handgrip strength was an independent predictor for OS in the multivariate analysis, but skeletal muscle depletion was not. Patients with low handgrip strength tended to discontinue lenvatinib therapy more often than patients with high handgrip strength (Figure 3A), while no significant difference was found between patients with and without muscle depletion (Figure 3B). A significant correlation was observed between the duration of TTF and OS time $(r=0.594$, $p<0.001$ ); furthermore, patients with low handgrip strength tended to discontinue lenvatinib therapy due to AEs compared with those with high handgrip strength ( $p=0.099$; data not shown).

Lenvatinib is recommended as a first-line treatment agent for advanced HCC [16-19]. In Japan, lenvatinib has been used as a second- or third-line intervention in real-world practice, after its approval in March 2018. A recent study demonstrated that initial lenvatinib therapy followed by conventional 
transcatheter arterial chemoembolization (CTACE) markedly improved OS compared with initial therapy of cTACE in patients with intermediate-stage HCC beyond up-to-seven criteria [30]. Moreover, the RESORCE study and the post-hoc analysis of the REFLECT study indicated that sequential therapy improved OS [20,31]. Therefore, using two or more MTAs administered sequentially is necessary to obtain a favorable OS for patients at intermediate and advanced stages of HCC. In addition, AE management should include administration of MTAs for a longer period. Some studies, including our study, have already reported that skeletal muscle depletion was associated with AEs, TTF, and OS in HCC patients treated with sorafenib [9-13]. However, to our knowledge, only one study reported the association between skeletal muscle mass and clinical outcome in HCC patients treated with lenvatinib [32].

Sarcopenia is defined as a progressive and generalized skeletal muscle disorder that involves reduction of muscle mass and functional decline [33]. The JSH proposed new diagnostic criteria, which could be used for Japanese patients with liver disease [5]. The JSH diagnostic criteria use identical cut-off values for skeletal muscle mass and grip strength; however, age-related criterion and assessment of physical performance such as walking speed, were not considered. Nagamatsu et al. reported that handgrip strength was associated with walking speed and all patients with slow walking speed showed low handgrip strength [34], and this report supports the JSH diagnostic criteria, which does not consider the walking speed. As our study population comprised of only Japanese patients, we adopted the JSH diagnostic criteria in this study. We investigated the individual effect of handgrip strength, skeletal muscle mass, and sarcopenia on clinical outcomes in HCC patients treated with lenvatinib.

In this study, we showed the effect of handgrip strength or sarcopenia on OS in HCC patients undergoing lenvatinib. However, skeletal muscle mass was not associated with OS. A large prospective cohort study and meta-analysis showed that low handgrip strength was significantly associated with the risk of cancer mortality $[35,36]$. However, only one study reported on the association between handgrip strength and mortality in patients with LC including HCC; the OS rates were significantly shorter in LC or HCC patients with low handgrip strength than in those with high handgrip strength, and low handgrip strength was independently associated with an increased risk of mortality, rather than skeletal muscle mass [37]. Although HCC patients were included, this report focused on all-cause mortality in LC patients.

As previous reports demonstrated that handgrip strength was associated with health-related quality of life and the incidence of composite hepatic events (e.g., hepatic decompensation and HCC, etc.) in patients with chronic liver diseases [38,39], patients with low handgrip strength might be more likely to experience AEs than those with high handgrip strength. Therefore, low handgrip strength might be also a predictor of AEs in HCC patients treated with lenvatinib.

In contrast, a recent study has reported that skeletal muscle depletion was associated with the occurrence of severe AEs, worse TTF, and poor OS in HCC patients treated with lenvatinib [32]. Some differences were noted in the clinical characteristics between our study and the previous study (our study: Child-Pugh B, 20.7\%; BCLC stage C, $47.2 \%$; presence of MVI, 20.8\%; presence of EHS, $28.3 \%$; presence of muscle depletion, $49.1 \%$; previous study: Child-Pugh B, $28 \%$; BCLC stage C, 51\%; presence of MVI, $27 \%$; presence of EHS, $36 \%$; and presence of muscle depletion, $59 \%$ ); liver function and HCC stage were worse in the previous study compared with those in our study. Consequently, the MST was shorter in the previous study (10.0 months) than in our study (24.0 months). Although skeletal muscle depletion was not an independent prognostic factor in our study, we considered that our result leaves room for reconsideration because of the short follow-up period. In addition, a long follow-up period is required.

Sarcopenia based on the JSH diagnostic guideline was associated with worse TTF and poor OS. Thus, it is possible to narrow the range of poor clinical outcome by means of this diagnostic guideline. However, the assessment of skeletal muscle mass using CT or BIA is limited in clinical practice because it is expensive, time-consuming, and involves radiation exposure. Measurement of handgrip strength, which can be performed even when at the bedside, is a simple, rapid, inexpensive assessment 
tool. Therefore, we considered that handgrip strength has a powerful effect on clinical outcomes of HCC patients treated with lenvatinib in real-world practice. A study reports that cancer rehabilitation was associated with prolonged survival in HCC patients treated with transcatheter arterial chemoembolization (TACE) [40] and cancer rehabilitation might also improve survival for HCC patients with low handgrip strength treated with lenvatinib. Further investigations are required on this topic.

This study had several limitations. First, this was a single-center retrospective cohort study assessing a small number of patients. Therefore, further multicenter studies with a larger population are warranted. Second, the follow-up period was short. Patients who discontinue lenvatinib therapy due to AEs or disease progression were treated with post-treatments (13 patients received sorafenib, then 6 of 13 received regorafenib. Four patients received hepatic arterial infusion chemotherapy and two of the four received sorafenib). At this time, the effect of these post-treatments on OS may not have been accurately assessed because of the short follow-up period. Moreover, a long follow-up period might draw a different conclusion regarding skeletal muscle mass.

\section{Conclusions}

Handgrip strength might be a useful marker of the clinical outcomes in HCC patients treated with lenvatinib. In addition, as handgrip strength measurement has an advantage over the assessment of skeletal muscle mass, it is well accepted in real-world clinical practice.

Supplementary Materials: The following are available online at http://www.mdpi.com/2076-3417/10/16/5403/s1, Table S1: Comparison of baseline characteristics in patients in handgrip strength or muscle depletion; Table S2: Comparison of baseline characteristics in patients with sarcopenia and non-sarcopenia.

Author Contributions: Y.K., I.S. (Issei Saeki), and T.Y. designed the study, analyzed the data, and wrote the paper. Y.K., I.S. (Issei Saeki), R.S., N.T., T.O., T.M. (Takashi Matsuda), T.H., T.M. (Toshihiko Matsumoto), I.H., and T.I. were involved in data acquisition. Y.K. and I.S. (Issei Saeki) performed the statistical analysis. T.T. and I.S. (Isao Sakaida) reviewed and amended the manuscript. All authors have read and agreed to the published version of the manuscript.

Funding: This research received no external funding.

Acknowledgments: We would like to thank Sanae Aritomi and staff of Division of medical nutrition at Yamaguchi University Hospital for their support and assistance with data collection. We would also like to thank Editage for English language editing.

Conflicts of Interest: The authors declare no conflict of interest.

\section{References}

1. Rosenberg, I.H. Summary comments: Epidemiological and methodological problems in determing nutritional status of older perosons. Am. J. Clin. Nutr. 1989, 50, 3. [CrossRef]

2. Rosenberg, I.H. Sarcopenia: Origins and clinical relevance. J. Nutr. 1997, 127, 990S-991S. [CrossRef] [PubMed]

3. Cruz-Jentoft, A.J.; Bahat, G.; Bauer, J.; Boirie, Y.; Bruyère, O.; Cederholm, T.; Cooper, C.; Landi, F.; Rolland, Y.; Sayer, A.A.; et al. Writing Group for the European Working Group on Sarcopenia in Older People 2 (EWGSOP2), and the Extended Group for EWGSOP2. Sarcopenia: Revised European consensus on definition and diagnosis. Age Ageing 2019, 48, 16-31. [CrossRef] [PubMed]

4. Chen, L.K.; Woo, J.; Assantachai, P.; Auyeung, T.W.; Chou, M.Y.; Iijima, K.; Jang, H.C.; Kang, L.; Kim, M.; Kim, S.; et al. Asian Working Group for Sarcopenia: 2019 Consensus Update on Sarcopenia Diagnosis and Treatment. J. Am. Med. Dir. Assoc. 2020, 21, 300-307. [CrossRef]

5. Nishikawa, H.; Shiraki, M.; Hiramatsu, A.; Moriya, K.; Hino, K.; Nishiguchi, S. Japan Society of Hepatology guidelines for sarcopenia in liver disease (1st edition): Recommendation from the working group for creation of sarcopenia assessment criteria. Hepatol. Res. 2016, 46, 951-963. [CrossRef] 
6. Hanai, T.; Shiraki, M.; Nishimura, K.; Ohnishi, S.; Imai, K.; Suetsugu, A.; Takai, K.; Shimizu, M.; Moriwaki, H. Sarcopenia impairs prognosis of patients with liver cirrhosis. Nutrition 2015, 31, 193-199. [CrossRef]

7. Meza-Junco, J.; Montano-Loza, A.J.; Baracos, V.E.; Prado, C.M.; Bain, V.G.; Beaumont, C.; Esfandiari, N.; Lieffers, J.R.; Sawyer, M.B. Sarcopenia as a prognostic index of nutritional status in concurrent cirrhosis and hepatocellular carcinoma. J. Clin. Gastroenterol. 2013, 47, 861-870. [CrossRef] [PubMed]

8. Fujiwara, N.; Nakagawa, H.; Kudo, Y.; Tateishi, R.; Taguri, M.; Watadani, T.; Nakagomi, R.; Kondo, M.; Nakatsuka, T.; Minami, T.; et al. Sarcopenia, intramuscular fat deposition, and visceral adiposity independently predict the outcomes of hepatocellular carcinoma. J. Hepatol. 2015, 63, 131-140. [CrossRef]

9. Imai, K.; Takai, K.; Hanai, T.; Ideta, T.; Miyazaki, T.; Kochi, T.; Suetsugu, A.; Shiraki, M.; Shimizu, M. Skeletal muscle depletion predicts the prognosis of patients with hepatocellular carcinoma treated with sorafenib. Int. J. Mol. Sci. 2015, 16, 9612-9624. [CrossRef]

10. Hiraoka, A.; Hirooka, M.; Koizumi, Y.; Izumoto, H.; Ueki, H.; Kaneto, M.; Kitahata, S.; Aibiki, T.; Tomida, H.; Miyamoto, Y.; et al. Muscle volume loss as a prognostic marker in hepatocellular carcinoma patients treated with sorafenib. Hepatol. Res. 2017, 47, 558-565. [CrossRef]

11. Nishikawa, H.; Nishijima, N.; Enomoto, H.; Sakamoto, A.; Nasu, A.; Komekado, H.; Nishimura, T.; Kita, R.; Kimura, T.; Iijima, H.; et al. Prognostic significance of sarcopenia in patients with hepatocellular carcinoma undergoing sorafenib therapy. Oncol. Lett. 2017, 14, 1637-1647. [CrossRef] [PubMed]

12. Saeki, I.; Yamasaki, T.; Maeda, M.; Kawano, R.; Hisanaga, T.; Iwamoto, T.; Matsumoto, T.; Hidaka, I.; Ishikawa, T.; Takami, T.; et al. No Muscle Depletion with High Visceral Fat as a Novel Beneficial Biomarker of Sorafenib for Hepatocellular Carcinoma. Liver Cancer 2018, 7, 359-371. [CrossRef]

13. Mir, O.; Coriat, R.; Blanchet, B.; Durand, J.P.; Boudou-Rouquette, P.; Michels, J.; Ropert, S.; Vidal, M.; Pol, S.; Chaussade, S.; et al. Sarcopenia predicts early dose-limiting toxicities and pharmacokinetics of sorafenib in patients with hepatocellular carcinoma. PLoS ONE 2012, 7, e37563. [CrossRef] [PubMed]

14. Kudo, M. Lenvatinib May Drastically Change the Treatment Landscape of Hepatocellular Carcinoma. Liver Cancer 2018, 7, 1-19. [CrossRef] [PubMed]

15. Kudo, M.; Finn, R.S.; Qin, S.; Han, K.H.; Ikeda, K.; Piscaglia, F.; Baron, A.; Park, J.W.; Han, G.; Jassem, J.; et al. Lenvatinib versus sorafenib in first-line treatment of patients with unresectable hepatocellular carcinoma: A randomised phase 3 non-inferiority trial. Lancet 2018, 391, 1163-1173. [CrossRef]

16. Marrero, J.A.; Kulik, L.M.; Sirlin, C.B.; Zhu, A.X.; Finn, R.S.; Abecassis, M.M.; Roberts, L.R.; Heimbach, J.K. Diagnosis, Staging, and Management of Hepatocellular Carcinoma: 2018 Practice Guidance by the American Association for the Study of Liver Diseases. Hepatology 2018, 68, 723-750. [CrossRef]

17. European Association for the Study of the Liver. EASL Clinical Practice Guidelines: Management of hepatocellular carcinoma. J. Hepatol. 2018, 69, 182-236. [CrossRef]

18. Kokudo, N.; Takemura, N.; Hasegawa, K.; Takayama, T.; Kubo, S.; Shimada, M.; Nagano, H.; Hatano, E.; Izumi, N.; Kaneko, S.; et al. Clinical practice guidelines for hepatocellular carcinoma: The Japan Society of Hepatology 2017 (4th JSH-HCC guidelines) 2019 update. Hepatol. Res. 2019, 49, 1109-1113. [CrossRef]

19. Korean Liver Cancer Association (KLCA); National Cancer Center (NCC). 2018 Korean Liver Cancer Association-National Cancer Center Korea Practice Guidelines for the Management of Hepatocellular Carcinoma. Korean J. Radiol. 2019, 20, 1042-1113. [CrossRef]

20. Alsina, A.; Kudo, M.; Vogel, A.; Cheng, A.L.; Tak, W.Y.; Ryoo, B.-Y.; Evans, T.R.J.; López López, C.; Daniele, B.; Misir, S.; et al. Effects of Subsequent Systemic Anticancer Medication Following First-Line Lenvatinib: A Post Hoc Responder Analysis from the Phase 3 REFLECT Study in Unresectable Hepatocellular Carcinoma. Liver Cancer 2020, 9, 93-104. [CrossRef]

21. Hiraoka, A.; Kumada, T.; Atsukawa, M.; Hirooka, M.; Tsuji, K.; Ishikawa, T.; Takaguchi, K.; Kariyama, K.; Itobayashi, E.; Tajiri, K.; et al. Real-life Practice Experts for HCC (RELPEC) Study Group, HCC 48 Group (hepatocellular carcinoma experts from 48 clinics in Japan). Prognostic factor of lenvatinib for unresectable hepatocellular carcinoma in real-world conditions-Multicenter analysis. Cancer Med. 2019, 8, 3719-3728. [CrossRef] [PubMed]

22. Tada, T.; Kumada, T.; Hiraoka, A.; Michitaka, K.; Atsukawa, M.; Hirooka, M.; Tsuji, K.; Ishikawa, T.; Takaguchi, K.; Kariyama, K.; et al. Neutrophil-to-lymphocyte ratio is associated with survival in patients with unresectable hepatocellular carcinoma treated with lenvatinib. Liver Int. 2020, 40, 968-976. [CrossRef] [PubMed] 
23. Takahashi, A.; Moriguchi, M.; Seko, Y.; Shima, T.; Mitsumoto, Y.; Takashima, H.; Kimura, H.; Fujii, H.; Ishikawa, H.; Takaharu, Y.; et al. Early Tumor Shrinkage as a Predictive Factor for Outcomes in Hepatocellular Carcinoma Patients Treated with Lenvatinib: A Multicenter Analysis. Cancers 2020, 12, 754. [CrossRef] [PubMed]

24. Tada, T.; Kumada, T.; Hiraoka, A.; Michitaka, K.; Atsukawa, M.; Hirooka, M.; Tsuji, K.; Ishikawa, T.; Takaguchi, K.; Kariyama, K.; et al. Real-life Practice Experts for HCC (RELPEC) Study Group and the HCC 48 Group (hepatocellular carcinoma experts from 48 clinics in Japan). Platelet-lymphocyte ratio predicts survival in patients with hepatocellular carcinoma who receive lenvatinib: An inverse probability weighting analysis. Eur. J. Gastroenterol. Hepatol. 2020. [CrossRef]

25. Shimose, S.; Kawaguchi, T.; Iwamoto, H.; Tanaka, M.; Miyazaki, K.; Ono, M.; Niizeki, T.; Shirono, T.; Okamura, S.; Nakano, M.; et al. Controlling Nutritional Status (CONUT) Score is Associated with Overall Survival in Patients with Unresectable Hepatocellular Carcinoma Treated with Lenvatinib: A Multicenter Cohort Study. Nutrients 2020, 12, 1076. [CrossRef] [PubMed]

26. Kirino, S.; Tsuchiya, K.; Kurosaki, M.; Kaneko, S.; Inada, K.; Yamashita, K.; Osawa, L.; Hayakawa, Y.; Sekiguchi, S.; Okada, M.; et al. Relative dose intensity over the first four weeks of lenvatinib therapy is a factor of favorable response and overall survival in patients with unresectable hepatocellular carcinoma. PLoS ONE 2020, 15, e0231828. [CrossRef]

27. Lencioni, R.; Llovet, J.M. Modified RECIST (mRECIST) assessment for hepatocellular carcinoma. Semin. Liver Dis. 2010, 30, 52-60. [CrossRef]

28. Hiraoka, A.; Michitaka, K.; Kumada, T.; Izumi, N.; Kadoya, M.; Kokudo, N.; Kubo, S.; Matsuyama, Y.; Nakashima, O.; Sakamoto, M.; et al. Validation and Potential of Albumin-Bilirubin Grade and Prognostication in a Nationwide Survey of 46,681 Hepatocellular Carcinoma Patients in Japan: The Need for a More Detailed Evaluation of Hepatic Function. Liver Cancer 2017, 6, 325-336. [CrossRef]

29. Forner, A.; Reig, M.; Bruix, J. Hepatocellular carcinoma. Lancet 2018, 391, 1301-1314. [CrossRef]

30. Kudo, M.; Ueshima, K.; Chan, S.; Minami, T.; Chishina, H.; Aoki, T.; Takita, M.; Hagiwara, S.; Minami, Y.; Ida, H.; et al. Lenvatinib as an Initial Treatment in Patients with Intermediate-Stage Hepatocellular Carcinoma Beyond Up-To-Seven Criteria and Child-Pugh A Liver Function: A Proof-Of-Concept Study. Cancers 2019, 11, 1084. [CrossRef]

31. Finn, R.S.; Merle, P.; Granito, A.; Huang, Y.H.; Bodoky, G.; Pracht, M.; Yokosuka, O.; Rosmorduc, O.; Gerolami, R.; Caparello, C.; et al. Outcomes of sequential treatment with sorafenib followed by regorafenib for HCC: Additional analyses from the phase III RESORCE trial. J. Hepatol. 2018, 69, 353-358. [CrossRef] [PubMed]

32. Uojima, H.; Chuma, M.; Tanaka, Y.; Hidaka, H.; Nakazawa, T.; Iwabuchi, S.; Kobayashi, S.; Hattori, N.; Ogushi, K.; Morimoto, M.; et al. Skeletal musle mass influences tolerability and prognosis in hepatocellular carcinoma patients treated with lenvatinib. Liver Cancer 2020, 9, 193-206. [CrossRef] [PubMed]

33. Cruz-Jentoft, A.J.; Sayer, A.A. Sarcopenia. Lancet 2019, 29, 2636-2646. [CrossRef]

34. Nagamatsu, A.; Kawaguchi, T.; Hirota, K.; Koya, S.; Tomita, M.; Hashida, R.; Kida, Y.; Narao, H.; Manako, Y.; Tanaka, D.; et al. Slow walking speed overlapped with low handgrip strength in chronic liver disease patients with hepatocellular carcinoma. Hepatol. Res. 2019, 49, 1427-1440. [CrossRef] [PubMed]

35. Celis-Morales, C.A.; Welsh, P.; Lyall, D.M.; Steell, L.; Petermann, F.; Anderson, J.; Iliodromiti, S.; Sillars, A.; Graham, N.; Mackay, D.F.; et al. Associations of grip strength with cardiovascular, respiratory, and cancer outcomes and all cause mortality: Prospective cohort study of half a million UK Biobank participants. BMJ 2018, 361, k1651. [CrossRef]

36. García-Hermoso, A.; Ramírez-Vélez, R.; Peterson, M.D.; Lobelo, F.; Cavero-Redondo, I.; Correa-Bautista, J.E.; Martínez-Vizcaíno, V. Handgrip and knee extension strength as predictors of cancer mortality: A systematic review and meta-analysis. Scand J. Med. Sci. Sports 2018, 28, 1852-1858. [CrossRef]

37. Hanai, T.; Shiraki, M.; Imai, K.; Suetsugu, A.; Takai, K.; Moriwaki, H.; Shimizu, M. Reduced handgrip strength is predictive of poor survival among patients with liver cirrhosis: A sex-stratified analysis. Hepatol. Res. 2019, 49, 1414-1426. [CrossRef]

38. Nishikawa, H.; Enomoto, H.; Yoh, K.; Iwata, Y.; Sakai, Y.; Kishino, K.; Ikeda, N.; Takashima, T.; Aizawa, N.; Takata, R.; et al. Health-related quality of life in chronic liver diseases: A strong impact of hand grip strength. J. Clin. Med. 2018, 7, 553. [CrossRef] 
39. Yoh, K.; Nishikawa, H.; Enomoto, H.; Iwata, Y.; Ikeda, N.; Aizawa, N.; Nishimura, T.; Iijima, H.; Nishiguchi, S. Grip strength: A useful marker for composite hepatic events in patients with chronic liver diseases. Diagnostics 2020, 10, 238. [CrossRef]

40. Hashida, R.; Kawaguchi, T.; Koya, S.; Hirota, K.; Goshima, N.; Yoshiyama, T.; Otsuka, T.; Bekki, M.; Iwanaga, S.; Nakano, D.; et al. Impact of cancer rehabilitation on the prognosis of patients with hepatocellular carcinoma. Oncol. Lett. 2020, 19, 2355-2367. [CrossRef] [PubMed]

(C) 2020 by the authors. Licensee MDPI, Basel, Switzerland. This article is an open access article distributed under the terms and conditions of the Creative Commons Attribution (CC BY) license (http://creativecommons.org/licenses/by/4.0/). 